\title{
Carnets
}

Revue électronique d'études françaises de l'APEF

Deuxième série - 2 | 2014

Frontières de la chronique

\section{Les chroniques parisiennes et politiques de Zola (1865-1872), au confluent de l'histoire, du journalisme et de la littérature}

\section{Claude Sabatier}

\section{OpenEdition}

Journals

Édition électronique

URL : http://journals.openedition.org/carnets/1331

DOI : 10.4000/carnets.1331

ISSN : 1646-7698

Éditeur

APEF

Référence électronique

Claude Sabatier, « Les chroniques parisiennes et politiques de Zola (1865-1872), au confluent de I'histoire, du journalisme et de la littérature », Carnets [En ligne], Deuxième série - 2 | 2014, mis en ligne le 30 novembre 2014, consulté le 30 avril 2019. URL : http://journals.openedition.org/carnets/1331 ; DOI : 10.4000/carnets.1331

Ce document a été généré automatiquement le 30 avril 2019.

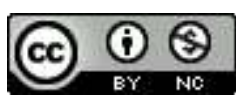

Carnets est mis à disposition selon les termes de la licence Creative Commons - Atribution - Pas d'utilisation commerciale 4.0 International. 


\title{
Les chroniques parisiennes et politiques de Zola (1865-1872), au confluent de l'histoire, du journalisme et de la littérature
}

\author{
Claude Sabatier
}

1 Travailler sur Émile Zola écrivain-journaliste relève d'une démarche modeste et ambitieuse, puisque, en-dehors des critiques d'art et des causeries littéraires, un corpus considérable de chroniques parisiennes et politiques est réuni sous le titre Chroniques et Polémiques I dans le volume 13 des Oeuvres Complètes de Zola au Cercle du Livre Précieux et dans les tomes 3 et 4 des éditions du Nouveau Monde. Ces articles balaient deux époques cruciales de notre Histoire, la fin du Second Empire et les débuts de la III ${ }^{\text {ème }}$ République, période-charnière de sept années de déliquescence politique, de flou institutionnel et de soubresauts guerriers ou insurrectionnels. Comment croiser et définir des genres ou des domaines aussi riches et interdépendants que l'histoire, la presse ou le roman, aux frontières de la chronique ? A cet égard, ce creuset journalistique nous semble allier à l'immédiateté insolite de l'actualité, à la périodicité dramatique des événements historiques, un jeu, une élaboration littéraires insoupçonnés dans cette partie moins connue de l'oeuvre zolienne.

De 1865 à 1872, Emile Zola, jeune Aixois ambitieux et révolté monté à Paris, fait ses gammes dans la petite presse et fourbit ses armes dans les publications politiques grâce aux contacts noués chez Hachette avec des écrivains, critiques et journalistes: collaborant à L'Événement illustré, mais surtout à La Tribune, au Rappel et à La Cloche, il répercute dans ses articles le mouvement d'opposition au Second Empire, qui, en se libéralisant en 1868, a signé son arrêt de mort; de même, avec l'avènement de la III ${ }^{\text {ème }}$ République en 1870, il se fait l'écho comme choniqueur parlementaire des Assemblées de Bordeaux, puis de Versailles, des hommes, des pratiques et débats de ces années de démocratie balbutiante. 
3 Par ailleurs, ses chroniques, tout en s'inscrivant dans la matrice médiatique de la presse du xix ${ }^{e}$ siècle (périodicité, collectivité, rubricité), offrent une grande variété de thèmes, de la mondanité apparente à la vie politique en passant par la flânerie ou la méditation sur la nature ou les commémorations, etc. Elles s'inscrivent aussi, selon la formule de Marie-Eve Thérenty dans La Littérature au quotidien, dans une "matrice littéraire" déployant un éventail impressionnant de formes (dialogues, discours, lettres...) et de registres (intimisme, ironie satirique, tentation pamphlétaire).

4 Enfin, plusieurs romans, La Curée, Nana, "romans de la morale ", La Conquête de Plassans, « roman de la religion », et Son Excellence Eugène Rougon, « roman de la politique », offrent des échos étonnants de ces articles, comme si le jeune journaliste avait testé dans ses chroniques des motifs, des situations, des personnages que le romancier réinvestira en les développant ou en les transposant: en somme, la presse aura été aussi la formule de Martin Kanes "l'atelier de Zola", un laboratoire du Grand Oeuvre zolien.

5 Toutefois, deux écueils se présentent face à cette hypothèse : d'une part, en l'absence de textes théoriques de Zola dans sa correspondance ou ses manuscrits, il est malaisé de définir des principes d'engendrement romanesque des éléments présents dans les chroniques; faute de traces avérées de réécriture des articles dans les romans, il faut tenter de caractériser « une fabrique journalistique du roman», pour parodier Colette Becker et sa Fabrique des Rougon-Macquart, d'épouser la fictionnalisation des chroniques, parfois potentiellement romanesques, telles ces Lettres de Paris de 1872 publiées dans les Nouveaux Contes à Ninon de 1874. D'autre part, on ne doit pas lire le texte romanesque dans une approche téléologique qui ne verrait dans la création littéraire que la mise en œuvre d'un programme déjà inscrit dans les chroniques mais montrer, au prix de multiples recroisements, des filiations possibles, des analogies troublantes, ou pour le moins une constance thématique et éthique.

6 Avant de rappeler dans quel contexte s'inscrivent les articles étudiés, définissons la chronique au sens historique du terme, puis dans son acception mondaine et populaire pour comprendre le sens que lui confère Zola. Recueil de faits historiques rapportés dans leur succession, la chronique médiévale vise à la fois à rendre compte du temps dans sa chronologie et à le capter dans son essence symbolique: Froissart, Villehardouin ou Joinville ne racontent pas seulement les croisades ou le règne de Saint Louis mais choisissent des moments typiques, répétitifs, admirables - construisant une légende ou une hagiographie. La pure succession postule donc aussi une exemplarité, une valeur emblématique.

7 A cette origine historique s'ajoutera une double acception journalistique, ainsi formulée dans le Larousse $d u$ XIX $x^{e}$ siècle : « article de journal où se trouvent les faits, les nouvelles du jour et les bruits de la ville", d'une part, la geste épique devenant, de tradition orale, rumeur - nouvelles vraies ou fausses, d'où la notion, péjorative, d'« échos », de chronique guettée par une superficialité dénoncée par maints... journalistes ; rubrique périodique, d'autre part, offrant une revue raisonnée, orientée de l'actualité dans un domaine donné. La chronique oscille donc entre l'écho littérairement virtuose d'une actualité anodine et l'article de fond où il s'agira moins de raconter tel événement que d'attendre l'échéance pour mesurer un changement, comprendre la portée du temps écoulé : aux antipodes de sa fonction première, rendre compte de la fuite des jours, la chronique va mesurer une modulation, la variation dans la répétition, et le prévisible ou la routine du cancan céder la place à l'insolite. 
Dans ses Lettres d'un curieux, Zola assigne à la chronique cette vocation à alerter et inquiéter les esprits. Ces deux textes inédits, écrits au printemps 1865 pour L'Avenir national, proposent un manifeste de la chronique, renouant, par-delà l'affadissement journalistique, avec la pureté originelle de cette notion. « Je sais que le mot chronique ne signifie pas autre chose que l'exposé des faits à mesure qu'ils se présentent. Changeons le mot alors pour changer la chose (...) Vous voulez un chroniqueur, et je ne puis vous offrir qu'un chroniqueur indiscipliné parfaitement ignorant des saisons et des fêtes » (Zola, Chroniques et Polémiques I, 1865: 45). A la curiosité mondaine et superficielle des chroniques élégantes et bourgeoises, l'auteur oppose donc la curiosité intellectuelle du moraliste et de l'historien. «Je tâcherai de ne surprendre que les vérités (...) En un mot, je suis décidé à causer longuement, chaque samedi, sur un unique sujet, à rire d'un travers contemporain, à applaudir ou à siffler le grand homme ou le grand événement du jour » ( ibid: 46). La chronique zolienne va ainsi d'autant mieux revêtir l'ampleur d'un éditorial que, loin de n'offrir qu'un constat, elle entend proposer une analyse des causes. Un homme de goût mis en scène et en abyme dans ce texte rêve ainsi :

Je voudrais qu'il se rencontrât un homme qui vit dans la chronique une revue satirique de notre société. Il résumerait en traits forts et rapides tous nos vices et toutes nos vertus (...) Il ne parlerait de tel fait que pour remonter à tel état d'esprit, il ne conterait les cancans que pour peindre les cancaniers. (ibid)

Curiosité intellectuelle et « scientifique », au sens naturaliste du terme :

Dès lors, nous aurions un chroniqueur dans la grande acception de ce mot (...) un moraliste qui nous conterait son temps, tirant la ficelle des personnages et faisant défiler le siècle devant nos yeux. Il écrirait les scènes détachées de la comédie, du drame contemporain; chacun de ses articles serait l'étude d'un des coins de notre société, et il pourrait ainsi, avec du temps et du courage, nous donner l'œuvre entière, l'histoire des hommes et des choses. (ibid : 46-47)

10 Ne croirait-on pas lire ici le projet fondateur des Rougon-Macquart dans la préface de $L a$ Fortune des Rougon?

11 Si les premiers articles de Zola remontent à 1863, dans Le Petit Journal, ses vrais débuts dans la presse, encouragés par son statut de chef de la publicité chez Hachette et de rédacteur de notices bibliographiques ou revues littéraires, peuvent être datés des Confidences d'une curieuse, publiées comme les Lettres d'un curieux en 1865, dans le Courrier $d u$ monde littéraire, artistique, industriel et financier d'Eugène Vattier. Commenter avec légèreté l'actualité étant la seule voie offerte au jeune journaliste en temps de censure impériale, la libéralisation de la presse n'arrivant qu'en 1867, Zola met en scène Pandore, une flâneuse curieuse et frivole distillant en anecdotes ou allégories des nouvelles mondaines, artistiques ou politiques : la première Confidence évoque ainsi l'enterrement du duc de Morny, la publication de L'Histoire de Jules César par...Napoléon III et la rentrée parlementaire du Sénat et du Corps législatif. Le personnage de Pandore permet de mimer la légèreté de la petite presse tout en satirisant le nivellement insipide de l'information, comme Timothée Trimm dans Le Petit Journal : masque du chroniqueur elle « donne rendez-vous à tous aux bains... d'Aix-en-Provence » et fait la promotion des Contes à Ninon - elle incarne le supposé badinage féminin et affecte la fausse naïveté d'un Persan ou d'un Huron.

Toute blanche et toute rose, riante et effarouchée, je me présente à vous, la boîte mystérieuse entre les mains (...) Il est faux que le coffret que me remit Jupiter ne contînt que des maux (...) que j'aie ouvert ce coffret avec la brusquerie d'une petite sotte qui dépense en un jour tous ses secrets (...) Je vous dirai quel fait imprévu s'en 
est échappé, de quelle plaie ou félicité j'ai doté la terre (Zola, Chroniques et Polémiques I, 1865 : 17). approche des vacances...), esquissent des thèmes préfigurant les articles donnés aux journaux républicains : préparatifs du Salon, médaille accordée au portrait de l'Empereur de Cabanel plutôt qu'à Corot et motif du mérite récompensé ou de l'Académie, à propos de l'élection du 6 avril... Savoureuses, les anecdotes parsèment les textes suivants: l'histoire du droguiste dupé par sa fille et son amant partis chercher à sa demande du " titi-la-ri-ton », herbe miraculeuse, celle de ce duc rejetant violemment sa maîtresse, humble portière qui a eu le mauvais goût de cirer ses chaussures - sans oublier l'apologue de «l'araignée monstre » et de "l'araignée buveuse d'huile » annonciatrices des personnages d'Archangias dans La Faute de l'abbé Mouret et Faujas dans La Conquête de Plassans. Le "coup d'épingle" se fait donc acéré pour la religion à l'approche de la semaine sainte mais aussi pour la Mode, « reine despotique qui force (...) tous les visages et toutes les tournures à s'accommoder de même ", avec « comme innovation, de l'Acier partout ».

13 Avec un hymne aux bois de Verrières apparait dans ces Confidences d'une Curieuse une autre veine, qui innervera les articles ultérieurs et fera même l'objet d'un véritable réinvestissement formel dans « Ce que disent les bois » paru le 12 avril 1870 dans La Cloche : la chronique-flânerie ou méditative, "esquisse de plein air» et «tentation du journalisme artiste » selon Henri Mitterand ${ }^{1}$, qui s'explique aussi par des considérations contextuelles: le vide de la saison, les impératifs de la censure ou les traditions de la périodicité médiatique. Le motif de la nature, prétexte à une caricature animalière de Sainte-Beuve et Guizot en grenouille et en scarabée recyclant les araignées monstre et buveuse d'huile, est subtilement lié à l'actualité politique, par la mise en abyme du journal comme objet emporté par habitude en promenade, symbole d'hétérogénéité textuelle et effet de réel dans son irruption brutale: ici l'assassinat de «ce grand citoyen ", Abraham Lincoln, là l'acquittement au procès de Tours de Pierre Bonaparte, assassin du journaliste républicain Victor Noir, dont « sanglote » la forêt...

des plaisants à une écriture plus conforme à son tempérament, ces neuf textes n'en aiguisent pas moins la verve satirique de Zola, ce regard acéré de moraliste sur le conformisme ou le mauvais goût, tout en esquissant les trois formes majeures de la chronique: chronique parisienne, chronique-promenade ou rêverie artiste, chronique politique...

Ces trois aspects caractériseraient assez bien également les 77 articles proposés par l'édition du Cercle du Livre Précieux et parus dans L'Événement illustré, La Tribune, Le Rappel, journal hugolien, et $L a$ Cloche, entre le 23 avril 1868 et le 17 août $1870: 26$ articles sur la mode, les spectacles, les récompenses ou le voyeurisme judiciaire paraissent dans L'Événement illustré; 31 textes, plus grinçants dans la dénonciation morale, politique et militaire, plus variés aussi sur le plan littéraire, figurent dans La Tribune; Le Rappel ne verra que 6 contributions de Zola, codées et symboliques, évoquant la déliquescence du Second Empire, le luxe insolent face à la misère, l'impunité politique et la caution cléricale pour masquer la désaffection du pays réel et de l'armée engagée dans un conflit aventureux ; enfin, 14 textes sarcastiques et allégoriques publiés dans La Cloche sonnent l'hallali du régime et d'une décadence morale et politique dont La Curée et Nana seront les romans.

Carnets, Deuxième série - 2 | 2014 

pauvreté, de la presse, du pouvoir impérial, de la religion et de l'armée. On compte 24 chroniques événementielles, artistiques, mondaines ou judiciaires, évoquant l'art, la littérature et le théâtre, le populisme et son pendant, le voyeurisme théâtral et surtout judiciaire, les femmes, liées pour Zola à la corruption et à l'artifice, les récompenses, caricatures du vrai mérite, l'éducation des jeunes filles et l'enseignement en général. En revanche, les chroniques-promenades, célébrant la nature, sont plus clairsemées. Ces thèmes ou genres sont souvent croisés, ainsi de la corruption qui relève d'un discours de satiriste sur les femmes, la mode et la coquetterie mais, plus profondément, résume pour le journaliste pamphlétaire l'essence même du régime honni.

elons la conjonction de trois facteurs : Zola, dans ces années 1868-1870, éprouvant des difficultés financières - il emprunte de l'argent à Manet - trouve dans la presse un moyen de subsister et d'exercer sa plume au moment où l'opposition républicaine, autour d'Eugène Pelletan ou de Théodore Duret, profite de la libéralisation de la presse - avec l'abolition de l'autorisation prélable - pour se doter d'un journal, La Tribune, machine de guerre préparant les élections de juillet 1869. Parallèlement, Zola entame la rédaction de ses Rougon-Macquart, avec le feuilleton de La Fortune des Rougon donné au Siècle. De cette interaction entre la presse et le roman autant que des circonstances politiques Zola tire un style incisif et virulent: "Il s'attaque (à cet Empire expirant) en se servant de la violence stylistique comme d'un instrument de dissection » explique Martin Kanes dans son Atelier de Zola'.

18

Cette collusion du talent et de la vie publique, des rencontres et des nécessités explique l'entrée de Zola au Rappel, créé par Charles Hugo et Henri Rochefort, le 4 mai 1869, en pleine campagne législative, et interdit avant même sa naissance! L'urgence du combat contre l'Empire fait oublier les oppositions esthétiques entre le libéralisme politique et le réalisme littéraire : Louis Ulbach, directeur de La Cloche, n'aimait guère Flaubert et l'on se souvient de sa dureté à l'encontre de Thérèse Raquin, « littérature putride » à ses yeux. Le Rappel, saisi et interdit de parution en juin 1869, la libéralisation de la presse remplaçant l'oppression par la persécution, se présentait comme un journal d'agitation, avec ses articles très découpés, tout en échos et potins, ses sous-entendus et dialogues satiriques, ses diatribes contre la censure, les communiqués gouvernementaux: son émiettement disparate offrait à l'apprenti journaliste une "matrice médiatique ${ }^{3}$ ", avec la collectivité du combat, la rubricité attrayante de sa présentation et la périodicité polémique de sa parution.

19 Si la collaboration de Zola au Rappel s'interrompt quelques mois, la radicalisation de l'opposition républicaine, après les grèves de mineurs, les incidents sanglants d'Aubin et l'assassinat du journaliste Victor Noir ramènent vite le journaliste dans le cercle hugolien : le numéro Les Soldats invoquant une armée républicaine détachée de l'Empire et les poursuites contre Rochefort lui inspireront la chronique «les vrais amis de l'armée » et l'acquittement de Pierre Bonaparte l'article cinglant «L'Empire est sauvé » évoquant le lâche soulagement des familiers de l'Empereur après les funérailles de Victor Noir. Le 3 février, "A quoi rêvent les pauvres filles ", construit en diptyque, oppose à la misère d'une jeune ouvrière dans son galetas le luxe insolent du pouvoir, exhibé par le procédé spéculaire du journal inséré que lit par hasard « la pauvre fille » : la description des toilettes au dernier bal des Tuileries, tirée du Figaro du 29 janvier 1870. Double effet de réel donc et promesse romanesque, puisque le même passage sera recyclé dans la description de la robe de Renée, au début de La Curée... 

de la radicalisation de Louis Ulbach, ou Ferragus, « ennemi absolu d'un régime qui blesse notre fierté, notre conscience et notre bon sens. » Avec ses rubriques impertinentes, « Les carillons parlementaires ", «La comédie politique » ou "La boîte aux calomnies », le quotidien dénonce inlassablement la répression de décembre 1851 contre la résistance au coup d'Etat: le feuilleton Les Bagnes de Cayenne, Souvenirs d'un transporté politique à la Guyane, de L. Pascal, exalte le «transporté » politique, qu'incarnera Florent dans Le Ventre de Paris ; quant à Arthur Ranc, futur Communard, il voit dans le plébiscite du 8 mai « un coup d'Etat permanen( $(t) »$. Zola publie jusqu'au 17 août 22 articles. Les premiers relèvent plus de la satire de mœurs et du cléricalisme mondain que de la polémique politique. réquisitoires de La Tribune pour dénoncer l'opulente richesse des profiteurs de la «curée » - le deuxième roman des Rougon-Macquart est en marche - et mettre en scène la «muette envie » du peuple. "Les Épaules de la marquise», parue le 21 février, se situe dans le même registre de la diatribe morale, en moins virulent, en plus ironique et allégorique, à l'image d' « A quoi rêvent les pauvres filles » du Rappel.

Les derniers articles témoignent de la hardiesse du journaliste dans le contexte de la guerre déclarée par l'Empereur le 17 juillet. Il n'hésite pas à prendre des risques, au point que le directeur, Louis Ulbach, doit le rappeler à la prudence pour éviter Mazas. L'inspiration zolienne passe du pamphlet moral à un discours violemment antibelliciste alors que les événements militaires sont censurés. Dans un texte intimiste et pathétique, il rêve, le 25 juillet, sur « Le Petit village », aujourd'hui inconnu, perdu sur le théâtre de la guerre, et dont « demain la rivière sera rouge ». Nouveau nom dans l'histoire, « il vivra éternellement dans les siècles, comme un coupe-gorge, un endroit louche où deux nations se seront égorgées. » L'avant-dernier article, « Vive la France », célèbre « sur les bords du Rhin cinquante mille soldats qui ont dit non à l'Empire », proclamant que " la République est là-bas, sur les bords du Rhin. » Pareils appels à la levée d'une armée républicaine contre la Prusse et... l'Empire et dénonciation de la récupération de La Marseillaise par «les petits crevés du journalisme officiel qui ont sali le patriotisme » auraient valu au journal et à son rédacteur des poursuites judiciaires sans la défaite de Wissembourg et la proclamation de l'état de siège. La Cloche interrompra sa publication le 18 août, au lendemain d'un dernier article de Zola «les Nerfs de la France», confiant dans la résistance face aux Prussiens. Le 11 août, le feuilleton de La Fortune des Rougon est suspendu.

Les débuts de la IIIème République vont offrir à Zola la matière d'articles pour Le Sémaphore de Marseille (Chemel, 1960-0961) mais surtout des nombreuses chroniques données à $\mathrm{L} a$ Cloche, dont il sera le correspondant parlementaire à Bordeaux puis Versailles; mais avant d'acquérir ce statut officiel, Zola part pour Marseille dès le 7 septembre. Il prend contact avec Émile Barlatier, directeur du Sémaphore de Marseille, et Alfred Arnaud, propriétaire du Messager de Provence. Profitant du climat insurrectionnel de la ville sous l'administration d'Esquiros, ancien confrère de La Tribune, il crée, avec l'aide de son ami aixois Marius Roux, le journal La Marseillaise, qui ne paraîtra que 2 mois et demi, du 27 septembre au 16 décembre. Seuls 6 articles de Zola (Ripoll, 1967) avaient été restitués et publiés par Le Messager de Provence jusqu'à ce qu'Adeline Wrona retrouve à la BNF des numéros de la collection complète (Wrona, 2011). Rejoignant à Bordeaux l'exécutif replié après l'évacuation de Tours, il devient secrétaire de Glais-Bizoin, son ancien directeur à La Tribune, membre du gouvernement de la Défense nationale. 
Les 6 articles de La Marseillaise, proposés par Patricia Carles et Béatrice Desgranges aux éditions du Nouveau Monde, frappent par leur véhémence patriotique et la dualité, réaffirmée, de Paris et de la province, autant de leitmotive de La République en marche: "La Marseillaise ", " Aux armes », "Le Deuil de Strasbourg ", après la chute symbolique du berceau de notre chant national, « Garibaldi ", « Paris compte sur la province », et « La Tête de Bismarck », caricature théâtrale du Machiavel prussien en machiniste sournois.

De février 1871 à août 1872, Zola, chroniqueur parlementaire, va donc donner à La Cloche mais aussi au Sémaphore de Marseille un nombre considérable d'articles, La République en marche. "Ses articles, rédigés à toute volée, donnaient à ses lecteurs un reportage coloré, dramatique, tantôt ému, tantôt sarcastique, toujours vécu, des discussions où se jouait le sort du pays » - explique Henri Mitterand ${ }^{4}$.

Ces articles se répartissent en grands ensembles chronologiques, dont la continuité ne sera interrompue que par la Commune et les vacances parlementaires. Une première série, intitulée Lettres de Bordeaux ${ }^{5}$, parut dans La Cloche du 13 février au 15 mars 1871.

De retour à Paris le 14 mars 1871, Zola reprend sa collaboration à La Cloche. Habitant rue La Condamine aux Batignolles, il doit prendre tous les jours le train pour Versailles, où siègera désormais l'Assemblée. Il n'assiste pas à la séance d'ouverture, le 20 mars, deux jours après la proclamation de la Commune, ayant été bloqué au moment où il s'apprêtait à quitter Paris mais rédigera un compte rendu de seconde main. Deux jours plus tard, le 22 mars, c'est un commissaire de Versailles qui le retient à la sortie de la gare. Ces contretemps, qui pour lui renvoient dos à dos Versaillais et Communards, n'interrompent pas son travail, qui prend fin le 18 avril avec la suspension de La Cloche par la Commune.

Parallèlement, la collaboration de Zola au Sémaphore de Marseille, du 25 avril au 7 juin, connue sous le titre de Lettres de Paris, palliant l'interdiction de La Cloche, offre un intérêt capital: elle nous offre des passages très durs sur la Commune, un point de vue si différent des chroniques de La Cloche qu'on a pu en contester l'attribution à Zola (Cogny, 1980). Rappelons brièvement que Zola, présent à Paris, au début de l'insurrection, dès le 14 mars, doit, face à une menace, réelle ou supposée, d'arrestation, partir pour SaintDenis le 10 mai puis Bonnières le 13 - «exil volontaire » que Rodolphe Walter évoque dans un article des Cahiers naturalistes. Malgré sa sympathie initiale pour les élections au Conseil de la Commune, au risque de déplaire à la direction de son journal, le journaliste exprime son rejet de la terreur contre le clergé ou les propriétaires, des perquisitions ou réquisitions, et du Comité de salut public, qui radicalise le mouvement, mené selon lui par «les factieux de l'Hôtel de Ville». Se sentant menacé d'arrestation à titre d'otage, il s'enfuit avec un passeport prussien : sa collaboration à La Cloche, suspendue, aurait déplu à Raoul Rigault, délégué de la Commune à la Sûreté générale, sans parler de la psychose obsidionale et de l'anonymat levé de sa correspondance au Sémaphore. Informé par courrier spécial et par les journaux parisiens ou versaillais qui lui parviennent, rentré le 26 mai à Paris, il évoquera la Semaine sanglante et déplorera pourtant avec une profonde compassion le massacre du peuple parisien (Ripoll, 1968).

Les nombreux articles de La République en marche pourraient se répartir autour de trois grandes thématiques : morale, économique et politique.

Pour la première source d'inspiration, les sujets abordés sont de deux ordres : sur le plan culturel, la défense d'une instruction laïque et notamment la nomination des instituteurs par l'Etat ou les communes; sur le plan religieux, les questions politiques de l'activisme clérical et du sort du pape, menacé par l'unité italienne et la politique ambiguë de 
Napoléon III, favorable aux nationalités et soucieux de se ménager les catholiques; l'atmosphère d'Ordre moral entretenue par les pantins de la droite dénoncés par Zola pour leur refus d'amnistier les Communards, leurs demandes de poursuites contre l'Internationale et leur moralisme clérical, sur le droit d'aînesse, l'alcoolisme ou les enfants naturels.

Un deuxième ensemble, plus vaste, de 42 articles, couvre les questions commerciales, financières et politico-juridiques : la question de l'emprunt, des indemnités de guerre ou pour les départements envahis, les problèmes récurrents à forte connotation politique (décentralisation et conseils généraux), ou, lors de la rentrée parlementaire de décembre 1871, les débats houleux entre Thiers et l'Assemblée sur le traité de commerce avec l'Angleterre, l'alternative entre impôt sur le revenu, rejeté par Thiers ou impôt sur les matières premières.

Les autres articles touchent à la politique proprement dite. Un certain nombre concerne le journalisme (les délits supposés de presse, les obstacles à sa diffusion); de longues chroniques évoquent la guerre, l'état de siège et surtout le traité de paix. Passionné ou critique, ennuyé ou désenchanté, témoigne des débats agitant l'Assemblée : querelles de partis sur la légitimité parlementaire des princes d'Orléans, séquelles du second Empire (pensions des magistrats de 1851, commissions mixtes, retour du bonapartiste Émile Rouher), mise en accusation par les monarchistes du 4 Septembre, des Communards (conseils de guerre, amnistie et commission des grâces, déportations) et surtout de l'Internationale; unité de la nation (statut communal de la capitale, opposition entre Paris et la province, siège de l'Assemblée); débats institutionnels, sur une Assemblée constituante et la nature du régime. Face à ces atermoiements procéduraux, à ce flou institutionnel, Zola, s'il condamne le bourreau des Communards, rend souvent hommage à la finesse politique de Thiers, qui, alliée à l'autorité d'un Eugène Rouher, lui inspireront le héros de Son Excellence Eugène Rougon. Une maxime subtilement paradoxale témoigne de cette fascination pour l'animal politique: "La prudence, chez lui, naît de la bataille même, et ses ténacités ont des accommodements inespérés. »

\section{***}

Venons-en à présent à la "matrice littéraire" dans laquelle se coulent ces textes, en nous appuyant sur les catégories envisagées par Marie-Ève Thérenty dans La Littérature au quotidien : la fictionnalisation, qui apparente, s'il en était besoin, maintes chroniques à des récits symboliques ou oniriques, le dialogisme, et l'intimisme, flânerie ou méditation.

Zola fictionnalise nombre de ses articles : fidèle à une esthétique narrative de la petite presse comme de la presse dite sérieuse, il s'amuse à mettre en scène en apologues ou anecdotes savoureuses des personnages réels ou fictifs, des situations plus ou moins oniriques, l'élaboration littéraire de ces "fictions militantes", selon l'expression de Corinne Saminadayar-Perrin, (Saminadayar-Perrin, 2009) lui offrant face à la censure un subtil jeu de masques, d'écrans, d'apologues ou d'allégories enchâssés, pris en charge par un narrateur second. La discussion sur l'impôt sur le revenu auquel s'oppose Thiers compensera sa technicité par ses multiples rebondissements, le chef de l'exécutif mettant sa démission dans la balance pour mieux la retirer une fois victorieux. De même, l'annonce du traité de paix semble en absorber le contenu dans la mise en scène d'une insupportable attente, distillée en un journal de bord éclaté et la dramatisation symbolique des valeurs bafouées, l'honneur et la patrie humiliés. A l'inverse, l'inanité 
d'un personnage, le baron Chaurand et de son interpellation prévue mais sans cesse différée, on ne sait sur quoi ce 28 février 1872, donne lieu à un psychodrame grotesque, à un récit faussement haletant qui s'achève par la mort du courrier épuisé annonçant tel Marathon un nouveau délai.

Ce tropisme narratif gagne les descriptions, ou portraits en action, telle l'évocation satirique d'un député monarchiste dont le sérieux ridicule déclenche des "essaims de rire » ici animalisés, comme si ses paroles ou actions étaient programmées dans son apparence de raideur :

On ne s'imagine pas quelle force comique il y a souvent au fond de la gravité d'un homme tel que M. Saint-Marc Girardin. Dans les pans de sa grande redingote noire, dans les plis de sa cravate blanche, des rires sont tapis, de petits rires folâtres qui s'envolent, par les beaux jours, comme des bandes de papillons. M. Saint-Marc Girardin a lâché l'essaim de ses rires (Zola, Chroniques et Polémiques I, lettre du 3 août 1872: 986)

Une modalité étonnante de la fiction journalistique est l'onirisme qui permet à la fois de dire l'ennui de certaines séances parlementaires et de frapper d'inanité dans une rêverie marine la dernière séance à Bordeaux, la «serre " parlementaire ou les prétentions fusionnelles des deux camps monarchistes, entourés de fées et feux follets. Le chroniqueur peut ainsi écrire :

Je m'imagine ces bons vieillards, tombés amoureux d'une vision qui a traversé le parc de Versailles, un soir, au crépuscule. C'est une apparition céleste, une fée blanche et rose, une ombre de joie et de ravissement (Zola, Chroniques et Polémiques I , lettre du 21 février $1872: 864$ ).

L'onirisme est doublement déprécié: en tant que tel, comme structure mentale des monarchistes et dans son contenu même, une impossible Restauration.

La chronique " Mardi gras: le rêve de Zola ", constitue un récit onirique, fondé sur l'allégorie et les métaphores filées du bal et du carnaval, où le rappel discret du statut journalistique de Zola sonne comme un retour à la réalité. «Je m’imaginais - rêve Zola sur le velours de ma loge, à l'Opéra, un samedi soir ». La séance de l'Assemblée devient fête du Mardi gras, le texte même vaste apologue où chaque personnage ou parti est codé, connoté. Masques, cotillons et faux nez envahissent le décor. Et quadrilles, menuets jusqu'à l'étonnante bourrée de $\mathrm{M}$. Rouher en bœuf gras - de se déployer avec les mille couleurs des déguisements : les monarchistes en chérubins, ours noirs ou hérissons, les radicaux, aux entrechats trop discrets, en forgerons ou revenants, sans oublier deux « communeux bien récalcitrants »... L'allégorie déploie satire et caricature.

Si l'article de presse prend souvent la forme de portraits ou récits, la mise en scène orchestrée conduit aussi, dans une intention pédagogique et littéraire, à créer des saynètes témoignant de la dimension dialogique du journal. En effet, le journal non seulement exhibe des discours parlementaires, des débats politiques, des conversations mondaines mais surtout postule un dialogue au jour le jour avec le lecteur et l'événement, dans l'instantanéité de l'article ou la périodicité de la chronique. L'appellation «Journal des débats» ou le titre même de «causerie» de nombre de chroniques sont symptomatiques de cette esthétique de proximité et de connivence avec le lecteur. Le dialogisme est une modalité irréductible au dialogue, revêtant aussi la forme du discours ou de la lettre.

Le discours occupe bien sûr une place de choix dans les chroniques parlementaires dont la fictionnalisation permet de contourner la technicité du propos ou d'éviter un compte 
rendu circonstancié par le récit de paroles, le renvoi au procès-verbal officiel - « Nous avons eu le discours de M. Thiers contre l'impôt sur le revenu. Je vous renvoie à ce document ", écrit ainsi le chroniqueur - la prétérition - " Je n'analyserai pas ce long discours, où l'orateur a répété ses premiers arguments " - voire la simple mention du discours, prétexte à un portrait satirique.

Le discours officiel lui paraissant souvent convenu ou fastidieux, Zola va déployer ses talents littéraires dans le discours fictif des chroniques impériales. La causerie parue dans La Tribune le 15 août 1869 est à cet égard symptomatique : elle évoque pour lui opposer un discours potentiel, moderniste et généreux, l'allocution convenue et passéiste sur la culture traditionnelle, l'érudition antique, prononcée par le ministre de l'Instruction publique, M. Bourbeau, lors de la distribution des prix du concours général à la Sorbonne. Le début du texte donne le ton : «Ah! Quel discours ! Un élève de rhétorique qui en aurait commis souvent de semblables n'aurait certes pas été appelé à entendre cette prose tiède et fade dont M. Bourbeau a noyé son auditoire (Zola, Chroniques et Polémiques I, lettre du 15 août 1869 : 237). » Le chroniqueur n'a pas de mots assez durs pour la médiocrité :

Et cependant, quelle admirable occasion pour parler avec tout son cœur et toute son intelligence! Un homme d'esprit supérieur, en face de ce jeune auditoire, frémissant d'espérance et de vie, trouverait, il me semble, de sublimes cris de tendresse (...) Le discours que je rêve ferait des hommes. L'auditoire entier se lèverait pour l'acclamer (...) En face de ces jeunes gens, l'orateur éprouverait une émotion poignante : il leur parlerait comme aux ouvriers qui achèveront d'établir la grande société du vingtième siècle. (Zola, Chroniques et Polémiques I, lettre du 15 août 1869 : 238).

On admirera la mise en scène du discours idéal, d'une utopie rhétorique où coïncideraient la générosité prophétique de l'idée et la séduction persuasive de la forme en des accents annonciateurs du grand œuvre des Rougon-Macquart. Curieux paradoxe d'un antidiscours, d'un discours rêvé qui sonne comme un manifeste de la pensée zolienne.

Raspail incarne ainsi, dans une émouvante chronique de La Cloche, en date du 14 juin 1870, "Les gaietés de la Chambre », cette parole pure, quasiment sacrée, humanitaire, face à la vanité des discours politiciens, au rire gras et grimaçant, topos romanesque au $\mathrm{XIX}^{\mathrm{e}}$ siècle :

$\mathrm{Au}$ milieu des rires, Raspail prononce cette phrase: "Celui dont vous riez, messieurs, a été élevé à l'école du malheur. J'ai vu les souffrances du prisonnier enfermé dans l'humidité, dans la saleté, sans même avoir un balai à sa disposition. » A ce mot de balai, vous comprenez, la Chambre n'y tient plus. Vous avez entendu: balai, il a dit : balai. Mon Dieu ! Que ce Raspail est cocasse. Il continue (...) : « Jamais, quant à moi, je ne ferai souffrir personne; quiconque souffre est un accusateur public. » Pas un de ces messieurs n'a l'air de se douter que ces paroles sont sublimes (Zola, Chroniques et Polémiques I, lettre du 14.06 .1870 : 297)

Notons enfin dans ce texte comment la parole journalistique oppose sa parole simplement fulgurante, en un aphorisme sarcarstique « C'est si comique, le cœur, l'émotion vraie ", à une intertextualité littéraire, où la référence culturelle est paradoxalement à la fois un marqueur négatif et l'arme de la satire anti-royaliste: "Jérôme David [en] Jupiter tonnant, M. Duvernois [en] demi-dieu qui escalade l'olympe. » Rappelons que nombre de discours émaillent les Rougon-Macquart, et, que face au discours utopique, un peu fumeux d'Etienne dans Germinal, dominent les discours officiels, verbeux et formalistes: inauguration à Niort du chemin de fer par Eugène au chapitre $\mathrm{X}$ de Son Excellence Eugène Rougon ou palinodie libérale du ministre à la fin du roman. 
la causerie «France, je te souhaite une année bonne et heureuse » sont fondées sur l'allégorie et le tutoiement de la France, les interpellations et les modalités de l'exhortation et de la prière. On pourrait citer la causerie adressée à Manon, exaltant une femme imaginaire, archétype pour Zola de la simplicité et du naturel, critique en creux de l'artifice, des spectacles poissards ou des femmes corrompues - bref, de cet univers glauque et chatoyant de La Curée ou Nana. La démarche épistolaire est ici nettement celle d'un moraliste pamphlétaire. Monologues insérés sur l'amour des champs et à l'inverse descriptions de fêtes mondaines émaillent cette lettre qui réussit à donner vie à un rêve : à l'illusion réaliste et familière du début - «Manon, ma chère, tu as raison; je te négligeais " - s'oppose l'effet de surprise et de démystification final: «Ah! que je t'aimerais, si tu existais, Manon, mon cher fantôme!» (Zola, Chroniques et Polémiques I, lettre du 27 septembre 1868, 188).

49 Un autre type de lettres adressées à l'ambassadeur de Chine, à Fatouma-Djombé, reine de Mohély, ou "A un prétendant » au trône de France, le roi d'Araucanie et de Patagonie, joue à la fois de la fantaisie exotique la plus débridée et du dépaysement fictif cher à Montesquieu, les rois d'opérette sollicités jouant le rôle du Persan voyageur tendant un miroir du second Empire et de l'esprit public. Plus personnalisée, «L'humble supplique » de Zola aux députés monarchistes préfère au dépaysement le jeu sur les registres : elle affecte l'imploration et la confidence pour masquer la véhémence du réquisitoire antimonarchique. L'ironie emprunte le masque du pathétique à Montesquieu dans sa célèbre « Très humble remontrance aux Inquisiteurs d'Espagne et de Portugal » de L'Esprit des Lois 
: Zola joue l'humilité et le respect du chroniqueur en priant ces royalistes lugubres, des « revenants », de rentrer à Paris. Il avance ensuite pour mieux les rejeter les prétextes qu'il pourrait invoquer : des anaphores déclinent à l'irréel du présent la forme refusée de cette supplique - «je pourrais, certes, vous dire que Paris est paisible»; «je pourrais encore vous parler des fêtes de l'Elysée »... « Telle est (...) l'humble supplique qu'ose vous adresser un ver de terre de la presse » (Zola, Chroniques et Polémiques I, lettre du 22 avril 1872, 950)

Un dernier ensemble de lettres est prêté à des personnages fictifs mais familiers, modestes politiques ou simples anonymes: un fusilier, un bonnetier, un député en tournée, le maire de Fouilly-les-Oies offrant l'hospitalité à "Sa Majesté champêtre " Napoléon III si l'Empereur venait à perdre le pouvoir, ou cet oncle frileux et conservateur rabrouant son neveu subversif. Ces lettres, dans les épistoliers desquels peut se reconnaître tout lecteur de l'époque, paraissent à la fois plus factuelles, car ancrées dans la réalité politique du régime, plus fictionnelles aussi car littérairement plus élaborées que les lettres allégoriques ou exotiques, où le dépaysement relevait parfois d'un jeu intertextuel et instaurait une distance atténuant la polémique.

51 Les chroniques zoliennes semblent ainsi à maints égards se couler dans la matrice de l'écriture intime. Elles revêtent une forme épistolaire dans maints articles tel le compte rendu du projet de traité devant l'Assemblée sous forme de journal intime ; elles relèvent enfin d'un compromis entre le nouveau régime de l'information fondé sur la primauté du fait et la tradition du journalisme littéraire, avec «la chose vue» et les chroniquesflâneries ou méditations.

L'écrivain-journaliste nous propose ainsi trois textes marquants: une chroniquepromenade à Gloton, hymne à la nature se déployant en éloge de la science, "épopée » (naturaliste) « de demain » et hommage aux poèmes de Michelet ; une causerie automnale sur les champs de foire de Saint-Ouen, qui permet à Zola d'exalter le peuple et ses plaisirs simples; une rêverie enfin sur les cimetières, méditation sur la mort et les lointains champs de bataille. Un même mouvement lyrique les anime, dépassant l'anecdote ou l'évocation en une morale, une visée symbolique: Zola semble ici essayer, avec la causerie, une forme originale de lyrisme critique ou d'argumentation poétique.

Zola nous propose une superbe causerie sur les cimetières, lieu romantique et mémoriel par excellence, qu'il affectionne (Zola, Chroniques et Polémiques I, lettre du 8 novembre 1868, 197-200). La chronique part d'une "rêverie de promeneur solitaire "; la rêverie, à son tour, s'essaie et se déploie en méditation, au fil des pensées, par associations d'idées rapprochements ou antithèses. «Le jour de la fête des Morts, j'ai suivi la foule, je suis allé, sous le pâle soleil de novembre, voir se fleurir les tombes » - écrit le promeneur, qui « (s'est) rendu lundi dernier au bord de la fosse commune ». Du plan d'ensemble Zola se focalise ensuite sur la partie déshéritée de nos nécropoles, la « fosse commune ». Par un nouvel élargissement s'ouvre une méditation dont les expressions «misérables petites croix noires » et «champ de misère » donnent la tonalité mélancolique : la médiation littéraire intervient ici, avec "une admirable page» de Germinie Lacerteux des frères Goncourt.

Dès lors, la causerie de Zola devient une magnifique déploration mortuaire, comme si elle trouvait seulement ici son vrai début. Zola en appelle à la compassion pour le peuple, à une digne sépulture : « Pourquoi ne pas laisser un peu de terre après leur mort à ceux qui n'ont rien possédé de leur vivant? Lundi dernier, j'ai vu des hommes en blouse, de pauvres femmes qui frissonnaient dans leur mince robe sombre...» Le tableau, enfin, 
s'individualise en une scène émouvante, narrative et dialoguée, entre le chroniqueur et une petite vieille :« comme j'avançais, j'ai aperçu une petite vieille qui errait tristement parmi les croix, regard(ant) le désespoir des autres avec des envies poignantes. » La petite vieille offre la vision pathétique d'une femme qui pleure ses trois fils, «l'un mort en Crimée, l'autre en Italie, le troisième au Mexique »: elle demande au chroniqueur la direction de Sébastopol, car elle ne sait pas même de quel côté se tourner pour prier pour son aîné ! Le lyrisme culmine en une déploration générale de la guerre dans une ultime vision, saisissante: «J'aurais voulu que chacun vit comme moi la pauvre petite vieille demandant de quel côté se trouvait Sébastopol et, priant les mains jointes, les yeux fixés sur le lointain horizon. » Déploration-réquisitoire contre le second Empire et ses morts sans sépulture...

Évoquons enfin la dimension pamphétaire qui anime maintes chroniques, notamment celles de la fin du second Empire, comme la causerie de La Tribune, du 29 août 1869, qui célèbre le devoir de mémoire face au coup d'Etat fondateur du régime à l'occasion de la parution du livre de Noël Blache Histoire de l'insurrection du Var en décembre 1851, matrice de La Fortune des Rougon. Voici l'ouverture, magistrale, de ce texte :

On a reproché à la démocratie son entêtement dans la haine. Selon certaines gens, les faits accomplis doivent être pardonnés (...) Je voudrais bien savoir sur quel calcul ces gens-là basent l'oubli des crises. Faut-il dix ans (...) pour qu'une mauvaise action devienne bonne? (...) La conscience humaine ne saurait avoir de ces compromis, et quand même une génération serait assez lâche pour oublier une date maudite, l'impartiale Histoire serait là, qui crierait à la postérité : «Tel jour, à telle heure, le droit a été violé et la France meurtrie » (Zola, Chroniques et Polémiques I, lettre du 29 août $1869: 244$ )

Ce préambule, riche en anaphores, antithèses et interrogations oratoires, ancre dans le registre du réquisitoire une chronique bien charpentée : présentation de Noël Blache, enfant interrogeant son grand-père devant l'arrestation de républicains au lendemain du coup d'Etat puis adulte devenu avocat, auteur d'une Histoire de l'insurrection du Var en décembre 1851 ; retour à une réflexion sur ce témoignage et la désinformation impériale présentant les républicains comme des assassins; enfin, larges citations du livre de $\mathrm{M}$. Blache, rapportant le meurtre de Besson, citations nourries d'un autre témoignage, Mémoires d'un fusillé, de Giraud, tisserand du Luc, qui reçut un coup de pistolet dans l'oreille de la part du gendarme Mayère. On sait comment ce drame sera recyclé à la fin de La Fortune des Rougon avec le meurtre de Silvère par Rengade.

Par la prolepse historique en appelant au jugement de la postérité, sa stigmatisation d'un adversaire manipulateur et inexpugnable, le régime et ses soutiens, son intransigeance morale au nom du "droit » et de la "conscience humaine ", ce superbe texte relève du pamphlet dont il illustrerait les trois caractères essentiels selon Marc Angenot dans La Parole pamphlétaire (Angenot, 1995): une vérité solitaire et véhémente, dont l'auteur indigné se sent dépositaire ; le sentiment par ailleurs d'une irrésistible imposture contre laquelle on en appelle à un auditoire universel - lecteurs ? postérité ? - pour combattre l'ennemi invisible ; enfin, dans une moindre mesure, tant Zola croit au progrès, une vision crépusculaire du monde, au moins dans la tonalité du texte.

La même virulence de prophète sacré semble animer deux textes dirigés l'un contre la décadence des mœurs sous le second Empire, l'autre contre l'Ordre moral qui exclurait les enfants naturels de l'exemption du service militaire, le moralisme aveugle du «fouet clérical» sensible dans la formule au style indirect libre «Sus aux enfants naturels. Ce 
sont des damnés» - monologue intérieur du baron? sarcasme du chroniqueur vitupérant ? - rejoignant l'immoralité débridée

Écoutez, voici où l'Empire en est venu, voici la génération qu'il a mise au monde (...) $C^{\prime}$ est du propre et le régal est complet (...) Voilà que nos hommes deviennent des femmes. Lorsque Rome pourrissait dans sa grandeur, elle n'a pas accompli d'autres miracles. Les belles nuits de l'orgie antique sont revenues, les nuits (...) où les créatures n'avaient plus de sexe (...) Ils devaient (...) en arriver là (Zola, Chroniques et Polémiques I, lettre du 13 février $1870: 261$ )

Ce texte, célèbre pour le motif de La Curée, est ainsi scandé par des formules ou interpellations vigoureuses qui célèbrent la vertu performative de la parole pamphlétaire, seule action possible contre le scandale et la perversion triomphante de la vérité. Mieux :

Ah! quelle curée que le second Empire! Dès le lendemain du coup d'Etat, l'orchestre a battu les premières mesures de la valse, et vite le thème langoureux est devenu un galop diabolique. Ils ont mis les mains aux plats, mangeant goulûment, s'arrachant les morceaux de la bouche (...) N'est-ce pas que la curée dure depuis d'assez longues années et qu'il est temps d'y mettre fin? (Zola, Chroniques et Polémiques I, lettre du 13 février $1870: 260$ )

Le motif de la curée est lié ici aux motifs du festin et du bal, qui l'inscrivent aussi dans un registre satirique, celle d'une ironie corrosive, jubilatoire et d'un monde inversé et carnavalesque, face auquel Zola semble hésiter entre une vision esthétique et une visée éthique, tel Gilquin, le personnage de Son Excellence Eugène Rougon entre bouffonnerie et basses œuvres.

61 Ainsi, l'articulation des chroniques et du cycle des Rougon-Macquart constitue pour Zola une rupture épistémologique apparentant la démarche journalistique à l'écriture romanesque : après des romans personnels, issus de son imagination, Thérèse Raquin ou Madeleine Férat, Zola combinera invention fictionnelle et satire morale et politique (issue du réservoir documentaire des chroniques), fondant la fiction sur l'analyse morale d'une société.

\section{BIBLIOGRAPHIE}

ANGENOT, Marc (1995). La Parole pamphlétaire. Typologie des discours modernes. Paris : Payot et Rivage.

CHEMEL, Henri (1960-1961). « Zola collaborateur du Sémaphore de Marseille » (1871-1877), Les Cahiers Naturalistes, $\mathrm{n}^{\circ} 14$, p. 555-567, $\mathrm{n}^{\circ} 18$, p. 71-79.

COGNY, Pierre (1980), "Le discours de Zola sur la Commune : étude d'un problème de réception », Les Cahiers Naturalistes, $\mathrm{n}^{\circ}$ 54, p. 17- 26.

KANES, Martin, (1963). Atelier de Zola, Textes de journaux (1865-1870). Genève : Droz.

MITTERAND, Henri (1962). Zola journaliste. Paris : Armand Colin

RIPOLL, Roger (1967). «Quelques articles retrouvés de La Marseillaise », Les Cahiers Naturalistes, n

- 34 , p. 148-164. 
RIPOLL, Roger (avril-mai 1968). « Zola et les Communards », Les Cahiers Naturalistes, n 35, p. 16-26. SAMINADAYAR-PERRIN, Corinne (2009). « " Lettres de Bordeaux " " : l'Histoire au jour le jour », Les Cahiers Naturalistes, $\mathrm{n}^{\circ} 83$, p. 116.

WRONA, Adeline (2011). Zola journaliste. Articles et chroniques. Paris : Garnier-Flammarion THÉRENTY, Marie-Eve (2007). La Littérature au quotidien. Poétiques journalistiques au XIXe siècle. Paris, Seuil, coll. « Poétique ». Paris : Seuil.

WALTER, Rodolphe (1972). « Zola et la Commune : un exil volontaire », Les Cahiers Naturalistes, $\mathrm{n}$ - 43, p. 25-37.

zolA, Émile (1865-1872). Cuvres Complètes. Tome treizième. Chroniques et Polémiques I, Confidences d'une curieuse, Chroniques, La République en marche. 1969, Paris : Cercle du Livre Précieux.

zolA, Émile (1868-1870). Cuvres Complètes. Tomes 3 et 4, "la naissance du naturalisme" - "la guerre et la Commune", 2003, Paris : Nouveau Monde éditions.

\section{NOTES}

1. Henri Mitterand, $1962: 36$.

2. Martin Kanes, $1963: 15$.

3. Marie-Eve Thérenty, $2007: 47$.

4. Émile Zola, 1865-72: 344 .

5. Zola y évoque la première séance de l'Assemblée nationale, la démission de Garibaldi, l'élection de Thiers à la tête du pouvoir exécutif, le désaccord croissant entre la province et Paris, le traité de Francfort, enfin l'annonce du transfert de l'Assemblée à Versailles, qui scelle le divorce entre Paris et la province, leitmotiv obsédant des chroniques.

\section{RÉSUMÉS}

De 1865 à 1872, Emile Zola fait ses gammes et fourbit ses armes dans la presse grâce aux contacts noués chez Hachette : collaborant aux journaux républicains, il épouse et répercute l'opposition au Second Empire ; de même, chroniqueur parlementaire à Bordeaux et Versailles de février 1871 à août 1872 , il témoigne de cette époque-charnière où la III ${ }^{\text {ème }}$ République peine à s'enraciner. Aux frontières de l'Histoire, de la presse et de la littérature, ses chroniques offrent une grande variété de thèmes, de l'actualité parisienne à la vie politique en passant par la flânerie méditative. Laboratoire du Grand Euvre zolien, elles déploient surtout un éventail impressionnant de formes littéraires et de registres, préparant des motifs ou des personnages que le romancier réinvestira dans La Curée, Nana, La Conquête de Plassans ou Son Excellence Eugène Rougon...

From 1865 to 1872, Emile Zola its ranges and prepares its defenses in the press through knotted Hachette contacts: Republicans working papers, he married and reflects opposition to the Second Empire ; Similarly, parliamentary chroniqueur Bordeaux and Versailles from February 1871 to August 1872, it reflects the time-hinge where the Third Republic only take root. The borders of 
history, the press and literature, his columns offer a wide variety of topics - news Parisian political life through meditative stroll. Laboratory of the Great Work zolien they mostly deploy an impressive array of literary forms and records, preparing patterns or characters that the novelist reinvest in The Quarry, Nana, The Conquest of Plassans or His Excellency Eugene Rougon...

INDEX

Mots-clés : chronique, pamphlet, presse, roman, satire

Keywords : chronic, pamphlet, newspaper, novel, satire

\section{AUTEUR}

\section{CLAUDE SABATIER}

Université Paris X - Ouest Nanterre La Défense

claudesabatier63[at]gmail.com 